\title{
Intrinsic optical bistability in a strongly driven Rydberg ensemble
}

\author{
Natalia R. de Melo, Christopher G. Wade, Nikola Šibalić, Jorge M. Kondo, Charles S. Adams, and Kevin J. Weatherill \\ Joint Quantum Centre (JQC) Durham-Newcastle, Department of Physics, Durham University, South Road, Durham DH1 3LE, UK
}

(Received 2 March 2016; published 30 June 2016)

\begin{abstract}
We observe and characterize intrinsic optical bistability in a dilute Rydberg vapor. The bistability is characterized by sharp jumps between states of low and high Rydberg occupancy with jump-up and -down positions displaying hysteresis depending on the direction in which the control parameter is changed. We find that the shift in frequency of the jump point scales with the fourth power of the principal quantum number. Also, the width of the hysteresis window increases with increasing principal quantum number, before reaching a peak and then closing again. The experimental results are consistent with predictions from a simple theoretical model based on semiclassical Maxwell-Bloch equations including the effects of interaction-induced broadening and level shifts. These results provide insight into the dynamics of driven dissipative systems.
\end{abstract}

DOI: 10.1103/PhysRevA.93.063863

\section{INTRODUCTION}

Optical bistability is a well-studied phenomenon that has provided a rich contribution to the understanding of nonequilibrium systems [1,2]. By definition, a system is bistable when, for the same input parameters, there are two stable output states. The classic conditions for observing optical bistability are a nonlinear system with feedback [3]. Different kinds of system have been used to demonstrate bistability such as Fabry-Perot cavities [3], nonlinear prisms [4], photonic crystal cavities [5], QED cavities [6], plasmonic nanostructures [7] and nematic liquid crystals [8]; and in general, for these examples, an optical cavity provides the feedback to the nonlinear system.

Intrinsic optical bistability occurs when a bistable response is present in a system without an optical cavity and feedback is replaced by strong interparticle interactions [9]. A dynamical equilibrium is reached between the driving of the excitation scheme and a dissipative process in the atom-light interaction. Recently, there has been much theoretical activity in exploring the dynamics of these driven-dissipative systems [2,10,11]. However, with observations to date limited to an up-conversion process using $\mathrm{Yb}^{3+}$ ions in a solid-state crystal [12] and more recently in strongly driven dilute Rydberg ensembles [13], there are few experimental studies that allow a detailed analysis of intrinsic optical bistability.

In this work, we extend the experimental and theoretical study of optical bistability in Rydberg ensembles [13] to investigate the behavior of optical bistability and hysteresis for a range of control parameters. By varying the atomic density, the excitation laser intensity and the principal quantum number $n$ of the Rydberg state we can control the driving and dissipation within the system. Our experimental observations reveal an increase in the hysteresis window width (or bistability width) followed by a subsequent narrowing as $n$ is increased.

\footnotetext{
*k.j.weatherill@durham.ac.uk

Published by the American Physical Society under the terms of the Creative Commons Attribution 3.0 License. Further distribution of this work must maintain attribution to the author(s) and the published article's title, journal citation, and DOI.
}

We also observe a saturation of the bistability width with driving laser intensity. Furthermore, the results are consistent with predictions from a surprisingly simple theoretical model based on the semiclassical Maxwell-Bloch equations including the effect of level shifts and broadening originating from additional processes in the Rydberg manifold.

\section{EXPERIMENT}

The atomic excitation scheme and a schematic of the experimental setup are shown in Figs. 1(a) and 1(b). The Rydberg state is accessed via resonant three-photon driving [14] by using a probe laser at $852 \mathrm{~nm}$ (Rabi frequency $\Omega_{\mathrm{p}}$ ) and a coupling laser at $1470 \mathrm{~nm}$ (Rabi frequency $\Omega_{\mathrm{c}}$ ) to drive the $\left|6 S_{1 / 2}, F=4\right\rangle \rightarrow\left|6 P_{3 / 2}, F^{\prime}=5\right\rangle$ and $\mid 6 P_{3 / 2}, F^{\prime}=$ $5\rangle \rightarrow\left|7 S_{1 / 2}, F^{\prime \prime}=4\right\rangle$ transitions, respectively. The probe and coupling beam $1 / e^{2}$ radius are 50 and $45 \mu \mathrm{m}$, respectively, and the beam powers are typically set in the range 20 to $70 \mu \mathrm{W}$. These lasers are stabilized on resonance by using polarization spectroscopy [15]. The final "Rydberg" laser (Rabi frequency $\Omega_{\mathrm{r}}$ ) is scanned across the resonance between $7 S_{1 / 2}$ and a Rydberg state $n P_{3 / 2}$. We access transitions to $n P_{3 / 2}$ states in the range $n=12$ to 50 , corresponding to a wavelength range from 882.70 to $780.03 \mathrm{~nm}$, using a Ti:sapphire laser. This laser has a $1 / e^{2}$ radius of $45 \mu \mathrm{m}$ and the typical power range is between $100 \mathrm{~mW}$ and $1 \mathrm{~W}$. All lasers are copropagating and focused through the center of a $2 \mathrm{~mm}$ Cs vapor cell. By using an interference filter, we reject the coupling and Rydberg light and measure the transmission of the probe beam. By means of population shelving in the Rydberg level [16] the transmission provides an effective readout of the population in the Rydberg state. The atomic density in the sample is controlled by varying the temperature of the cell [17], which is dynamically stabilized.

Figure 2(a) shows the transmission of the probe beam as a function of the Rydberg laser detuning $\Delta$, sweeping the frequency in positive (red) and negative (black) directions. Each curve corresponds to the spectrum for a different $n P_{3 / 2}$ Rydberg state with $n=34,35$, and 37 . The results were obtained at atomic density of $(1.0 \pm 0.5) \times 10^{10} \mathrm{~cm}^{-3}$, with probe and coupling Rabi frequency, $\Omega_{\mathrm{p}} / 2 \pi=(140 \pm 10)$ and $\Omega_{\mathrm{c}} / 2 \pi=(170 \pm 10) \mathrm{MHz}$. The Rabi frequency of the final step transition is kept constant at $\Omega_{\mathrm{r}} / 2 \pi=(80 \pm 10) \mathrm{MHz}$ as $n$ 
(a)
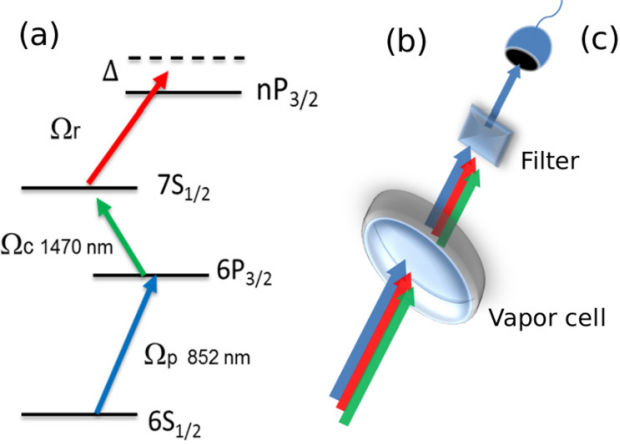

(c)

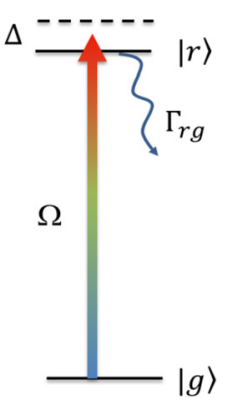

FIG. 1. (a) Three-photon excitation scheme to Rydberg states in cesium. (b) Schematic of experimental setup. The three excitation lasers copropagate through a $2 \mathrm{~mm}$ vapor cell. An interference filter is used to selected the transmission signal of the probe beam. (c) Simplified two-level model system.

is changed. Under these conditions we observe an asymmetric lineshape with a sharp phase transition between the states of low and high Rydberg occupancy, shifted to the red side of resonance.

The behavior of the measured shift as a function of $n$ is shown in Fig. 2(b). The frequency shift of the phase transition is seen to scale with the fourth power of the principal quantum number $\left(\approx n^{4}\right)$, as we show with the solid (red) line fit. This result is consistent with an interaction shift due the strong dipole-dipole interactions between Rydberg atoms [18], since the interaction potential between two dipoles is $V_{\mathrm{dd}} \propto d^{2}$ where dipole moment $d \propto n^{2}$. However, other interactions, such as ionizing collisions [19] or dipole-dipole energy transfer [20] could have the same $n$ scaling.

Upon stronger driving or increased atomic density, above a critical limit, the behavior becomes more complex. The interaction-induced frequency shift surpasses the width of the optical resonance, which is dominated by the Rabi driving of the first two transitions, $\Omega_{\mathrm{p}}$ and $\Omega_{\mathrm{c}}$, resulting in an
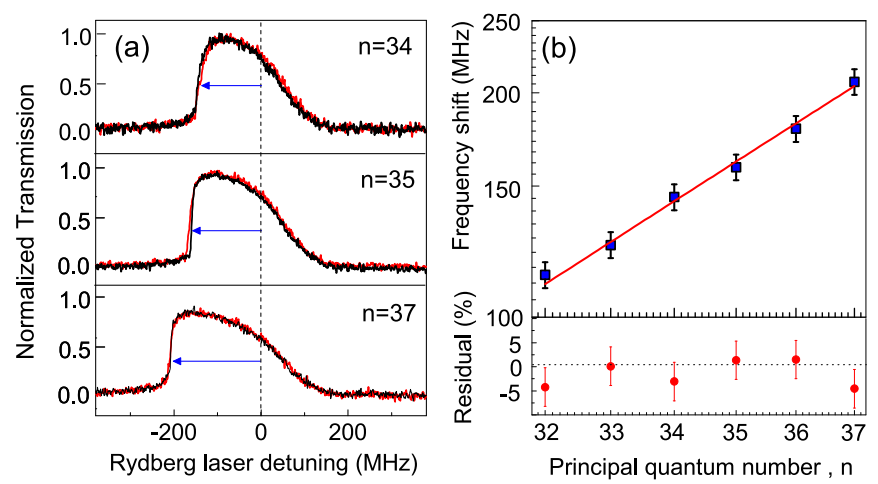

FIG. 2. (a) Experimental optical response, the transmission of the probe beam as a function of the Rydberg laser detuning for the same Rabi frequency $\Omega_{\mathrm{r}} / 2 \pi=(80 \pm 10) \mathrm{MHz}$ and different Rydberg levels $n=34$, 35, and 37. $\Omega_{\mathrm{p}} / 2 \pi=(140 \pm 10)$ and $\Omega_{\mathrm{c}} / 2 \pi=$ (170 \pm 10$)$ MHz. (b) Measurement of the frequency shift of the phase transition as a function of principal quantum number $n$, of the Rydberg level $n P_{3 / 2}$. The solid (red) line is a fit showing that the shift scales with $(n-\delta)^{\alpha}$, where $\delta$ is the quantum defect of the Rydberg state $n P_{3 / 2}$ and $\alpha=3.8 \pm 0.3$.
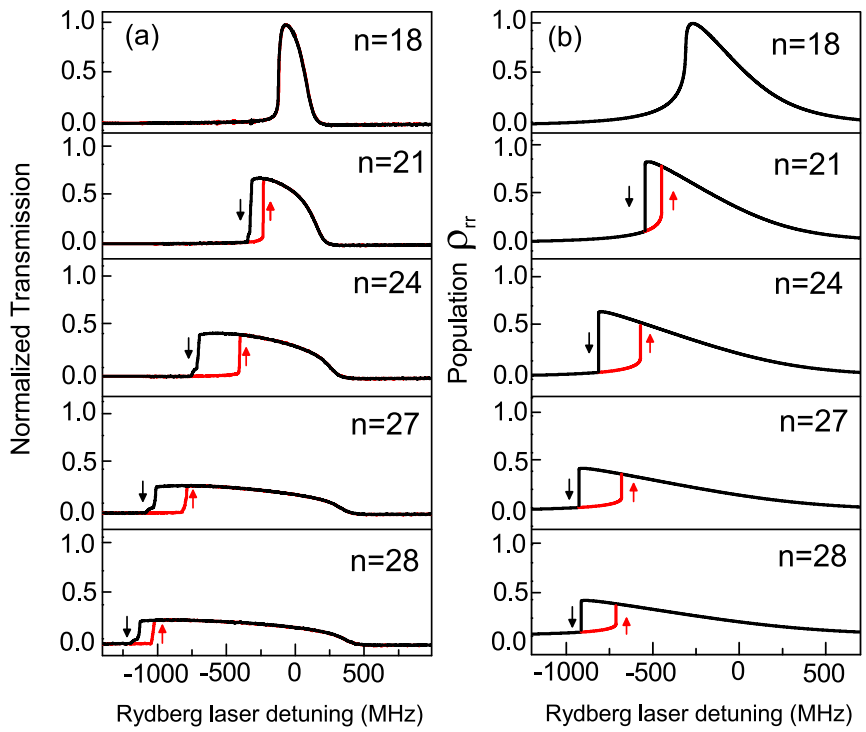

FIG. 3. (a) Experimental optical response; the transmission of the probe beam as a function of the Rydberg laser detuning for the same Rabi frequency $\Omega_{\mathrm{r}} / 2 \pi=(130 \pm 10) \mathrm{MHz}$ and different Rydberg levels. (b) Theoretical Rydberg-state population $\rho_{\mathrm{rr}}$ from the model as a function of the laser detuning for different Rydberg levels. Theoretical parameters are $\Omega_{\mathrm{r}} / 2 \pi=40 \mathrm{MHz}$ and $\Gamma_{\mathrm{rg}} / 2 \pi=3 \mathrm{MHz}$.

intrinsic optical bistability and hysteresis. Figure 3(a) shows the transmission of the probe beam as a function of the Rydberg laser detuning for different Rydberg states, ranging from $n=18$ (top) to $n=28$ (bottom). This result is obtained at atomic density of $N=(3.0 \pm 0.5) \times 10^{11} \mathrm{~cm}^{-3}$ and Rabi frequencies $\Omega_{\mathrm{p}} / 2 \pi=(130 \pm 10), \Omega_{\mathrm{c}} / 2 \pi=(190 \pm 10)$ and $\Omega_{\mathrm{r}} / 2 \pi=(130 \pm 10) \mathrm{MHz}$. Each curve shows positive (red) and negative (black) scan across the resonance for different Rydberg levels and are normalized to the peak transmission amplitude of the $n=18$ state. With the exception of the $n=18$ state, each scan displays obvious optical bistability with hysteresis dependent on the direction in which resonance is approached, indicated by arrows on the plots. Within the hysteresis region the system can be maintained in two different steady states of high and low Rydberg occupancy for the same experimental parameters. As $n$ is increased the width of the hysteresis window initially becomes larger followed by a subsequent narrowing. The evolution of the bistability width with $n$ is shown in Fig. 4 for two different atomic densities $N=$ $(2.0 \pm 0.5) \times 10^{11}$ (purple squares) and $(3.0 \pm 0.5) \times 10^{11} \mathrm{~cm}^{-3}$ (green circles). The range of $n$ accessed is ultimately limited by the maximum power of the Rydberg laser to keep the Rabi frequency $\Omega_{\mathrm{r}}$ constant. For low atomic density we observe a saturation of the bistability width for higher $n$, but for high atomic density we can observe a maximum value at $n=26$ and subsequent narrowing.

\section{THEORETICAL MODEL}

To understand the bistability we model the system by using the density-matrix formalism applied to a simple two-level system, as shown in Fig. 1(c). We consider a ground state $|g\rangle$ and a Rydberg state $|r\rangle$ coupled by a laser with Rabi frequency 


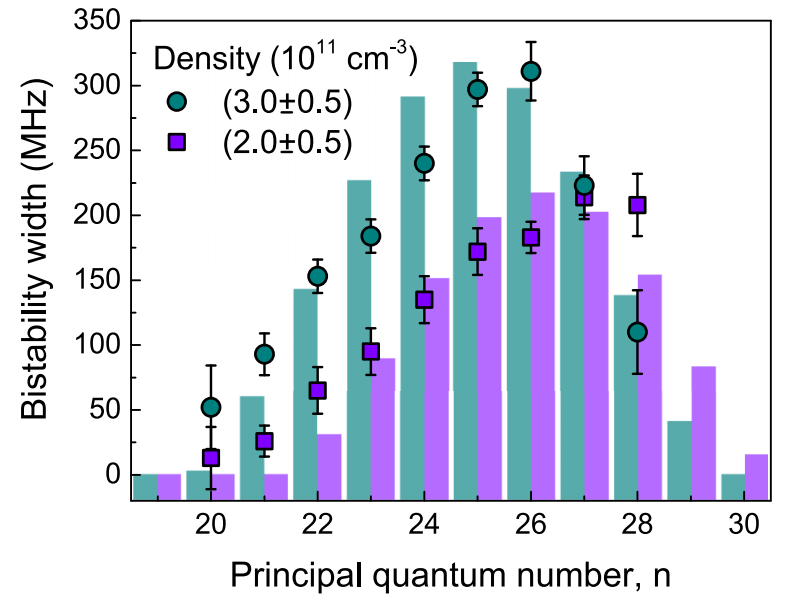

FIG. 4. Bistability width as a function of the principal quantum number for two atomic densities, $N=(2.0 \pm 0.5) \mathrm{cm}^{-3}$ (purple squares) and $(3.0 \pm 0.5) \times 10^{11} \mathrm{~cm}^{-3}$ (green circles), for the same Rydberg Rabi frequency, $\Omega_{\mathrm{r}} / 2 \pi=(120 \pm 10) \mathrm{MHz}$. The column bars are the theoretical results considering the interactionbroadening effect, $\beta^{\prime} / 2 \pi=(2.0 \pm 0.2) \times 10^{-9} \mathrm{~Hz} \mathrm{~cm}^{3}$ and the parameters $\Gamma_{\mathrm{rg}} / 2 \pi=13.5 \mathrm{MHz}$ and $\Omega / 2 \pi=(140 \pm 5) \mathrm{MHz}$.

$\Omega$ and detuning $\Delta$, where $\Gamma_{\mathrm{rg}}$ is the width of the optical resonance. Using semiclassical analysis, the time evolution of the system is described by a Lindblad master equation applied to a simple two-level system [21]. We use mean-field theory to describe the dipole-dipole interaction between the Rydberg atoms in a classical approximation [22], where the many-body interaction is described in terms of the response of a single atom interacting with a mean-field interaction potential. This results in a renormalization of the transition frequency $\Delta \rightarrow \Delta_{\text {eff }}=\Delta-\Delta_{\text {dd }}$, where $\Delta_{\text {eff }}$ is an effective detuning [22] and $\Delta_{\mathrm{dd}}$ is the mean-field shift which can be expressed as $\Delta_{\mathrm{dd}}=V \times \rho_{\mathrm{rr}}$, where $\rho_{\mathrm{rr}}$ is the fraction of atomic population in the Rydberg state and $V$ is the interaction term corresponding to the sum of the dipole-dipole interaction over the excitation volume.

We include the renormalization in the optical Bloch equations (OBEs), giving the time evolution of the matrix elements as

$$
\begin{gathered}
\dot{\rho_{\mathrm{gr}}}=i \Omega\left(\rho_{\mathrm{rr}}-1 / 2\right)+i\left(\Delta-\mathrm{V} \rho_{\mathrm{rr}}\right) \rho_{\mathrm{gr}}-\frac{\Gamma_{\mathrm{rg}}}{2} \rho_{\mathrm{gr}}, \\
\dot{\rho} \mathrm{rr}=-\Omega \operatorname{Im}\left(\rho_{\mathrm{gr}}\right)-\Gamma_{\mathrm{rg}} \rho_{\mathrm{rr}},
\end{gathered}
$$

where the fractions of the atomic population in the ground and Rydberg states, $\rho_{\mathrm{gg}}$ and $\rho_{\mathrm{rr}}$, respectively, are related as $\rho_{\mathrm{gg}}=1-\rho_{\mathrm{rr}}$, and the coherence terms are related as $\rho_{\mathrm{gr}}=\rho_{\mathrm{rg}}^{*}$. The steady-state solution can be found by assuming $\dot{\rho}_{\mathrm{gr}}=$ $\dot{\rho}_{\text {rr }}=0$ and solving the set of equations (1). We obtain a cubic equation for the population in the Rydberg state:

$$
\frac{\Omega^{2}}{4}-\left(\frac{\Omega^{2}}{2}+\frac{\Gamma_{\mathrm{rg}}^{2}}{4}+\Delta^{2}\right) \rho_{\mathrm{rr}}-2 \Delta V \rho_{\mathrm{rr}}^{2}-V^{2} \rho_{\mathrm{rr}}^{3}=0 .
$$

This equation provides the steady-state solution for the Rydberg population as a function of the Rydberg-laser detuning.
The basic model described so far can explain the origin of the optical bistability in the experimental system as shown in Ref. [13]. As $V$ increases from zero the model predicts an asymmetrical lineshape, similar to those presented in Fig. 2(a). We link the shift measured in Fig. 2(a) to the parameter $V$ because the model predicts the position of the greatest slope is proportional to the parameter $V$ (in the limit $\Omega \gg \Gamma_{r g}$ ). On the strength of this relation we introduce a scaling of $V \propto$ $n^{* 4}$, where $n^{*}$ is the effective principal quantum number. As $V$ increases further a hysteresis window opens and we see three distinct solutions. One solution is unstable, but the other solutions correspond to the two stable steady states of the bistable regime.

While this initial model incorporates bistability, it does not capture the closing of the bistability at high density or principle quantum number seen in Fig. 3(a). We therefore extend our model to include the effect of an interaction-broadening contribution where the total relaxation rate is rewritten as

$$
\Gamma_{\mathrm{rg}} \rightarrow \Gamma=\Gamma_{\mathrm{rg}}+\Gamma_{\mathrm{int}}=\Gamma_{\mathrm{rg}}+\beta N,
$$

where $\beta$ is the interaction-broadening coefficient and $N$ is the atomic density. For simplicity, we apply the same $n^{4}$ scaling observed for the frequency shift to the interaction-broadening term and write the interaction-broadening coefficient as

$$
\Gamma_{\text {int }}=\beta^{\prime}(n-\delta)^{4} \mathrm{~N},
$$

where $\delta$ is the quantum defect of the Rydberg state $n P_{3 / 2}$, which the value we use as 3.55 [23].

In our system different mechanisms may contribute to the interaction-broadening effect, such as associative ionization [24] or photoionization [23], and different collisional process such as state mixing [25,26], but we are not able to distinguish each individual contribution. Self-broadening coefficients have been studied in atomic vapors for many years particularly for the $D 2$ lines of alkali-metal atoms $[27,28]$. However, in this work on Rydberg states, we expect the broadening to have a number of different contributions. We note that our model does not explicitly include the multilevel structure of the atom, Doppler effects, and the effects of absorption of the laser beams through the vapor; such a detailed model is beyond the scope of this work. However, we motivate our simple model through its qualitative and quantitative resemblance to our experimental results.

\section{ANALYSIS AND DISCUSSION}

In Fig. 3(b) we present the results of our model and plot Rydberg-state population $\rho_{\mathrm{rr}}$ as a function of laser detuning for several Rydberg levels. To compare these results with the experimental data plotted in Fig. 3(a), the curves are normalized to the maximum transmission amplitude of the $n=18$ scan. The addition of the broadening term to the model is essential to reproduce the bistability window closing at higher principal quantum number. The results show good qualitative agreement with respect to the amplitude of the transmission signal and the width of the hysteresis window. For this calculation, the theoretical parameters are $\Omega / 2 \pi=$ $40 \mathrm{MHz}$ and $\Gamma_{\mathrm{rg}} / 2 \pi=3 \mathrm{MHz} . \Gamma_{\mathrm{rg}}$ is influenced by several competing factors. On one side there is slow decay from Rydberg states $(<1 \mathrm{MHz})$ and on the other side there is saturation broadening from the Rabi driving of the first two 
transitions ( $>100 \mathrm{MHz})$. The simple model cannot capture all of this behavior, so $\Gamma_{\mathrm{rg}}$ should be seen as a phenomenological parameter which does not have a direct physical interpretation.

In Fig. 4, a quantitative comparison is shown for the bistability width as a function of the principal quantum number for two atomic densities, $N=(2.0 \pm 0.5)$ and $(3.0 \pm$ $0.5) \times 10^{11} \mathrm{~cm}^{-3}$. The full circles and squares with the error bars are the experimental data, and the column bars are the theoretical-fit results including the interaction-broadening effect. Bistability is typically present in the model when $V>\Omega>\Gamma$, and so as the principle quantum number is increased, the bistability window opens when $V>\Omega$ and starts to close again when $\Gamma>\Omega$. Although we find good numerical agreement for the bistability width, we note that the overall detuning of the bistability window was smaller than predicted by the model.

By fitting the low-density data in Fig. 4 (purple squares) we find that the ratio $\Omega / \Omega_{\mathrm{r}} \approx 1$ which is expected because the transition of the two first excitation steps are saturated. The discrepancy with the ratio $\Omega / \Omega_{\mathrm{r}} \approx 0.3$ used in Fig. 3 is because we are now only fitting the bistability width and disregarding the overall shift. The parameter $V$ is constrained to follow $n^{* 4}$ scaling and we find a value $V=\left(8.9 \times 10^{-8}\right) n^{* 4} N \mathrm{~Hz} \mathrm{~cm}^{3}$, which is used consistently for all the theoretical calculations of this work. For the interaction-broadening coefficient, we get a value $\beta^{\prime} / 2 \pi=(2.0 \pm 0.2) \times 10^{-9} \mathrm{~Hz} \mathrm{~cm}^{3}$, which is a key quantitative result to characterize the system.

Many previous studies of line-broadening effects were performed with lower atomic states $[28,29]$ in contrast to Rydberg states where the strong interactions and multiple contributions to the broadening term is a distinguishing characteristic $[23,30]$. We compare our result for $\beta$, with the self-broadening coefficient for cesium Rydberg states, $\gamma_{\text {self }}$, which has been measured directly [31]. Self-broadening only originates from interactions between excited-state atoms and the surrounding ground-state atoms. For the parameters of interest $(20 \leq n \leq 28), \gamma_{\text {self }}$ does not scale monotonically with $n$ but rather exhibits a series of resonances, reaching a plateau for $n>30$, with $\gamma_{\text {self }} / N=(3.3 \pm 0.6) \times 10^{-7} \mathrm{~Hz} \mathrm{~cm}^{-3}$. If we compare $\gamma_{\text {self }}$ with our value of $\beta=\beta^{\prime}(n-\delta)^{4}$ (for $n=28$ ) we find that the self-broadening coefficient is three orders of magnitude too small to explain our result. This is a strong indication that the physical origin of our broadening term comes from interactions within the Rydberg manifold.

Finally, we characterize the width of the bistability window for a fixed Rydberg state $(n=28)$ as a function of Rydberg laser power for three different atomic densities shown in Fig. 5: $N=\{3.0(\mathrm{O}) ; 1.0(\square) ; 0.7(\triangle)\} \pm 0.5 \times 10^{11} \mathrm{~cm}^{-3}$. The solid lines represent the theoretical calculation for the each density and the Rabi frequency is related to the Rydberg laser power $P$ by $\Omega \propto \sqrt{P}$. By finding the best fit of the model to the data, we find $\Omega / 2 \pi=(137 \pm 2) \sqrt{P} \mathrm{MHz}$ which confirms the ratio $\Omega / \Omega_{\mathrm{r}} \approx 1$ as $\Omega_{\mathrm{r}} / 2 \pi=(130 \pm 10) \sqrt{P} \mathrm{MHz}$ for the experimental data. The fitted value of the interactionbroadening coefficient, $\beta^{\prime} / 2 \pi=(1.6 \pm 0.2) \times 10^{-9} \mathrm{~Hz} \mathrm{~cm}^{3}$, is consistent with the value found in the best fit for the data in Fig. 4.

In Fig. 5 the bistability width saturates as the power broadening of the optical resonance catches up with the interaction energy $V$. At low density, this happens with smaller

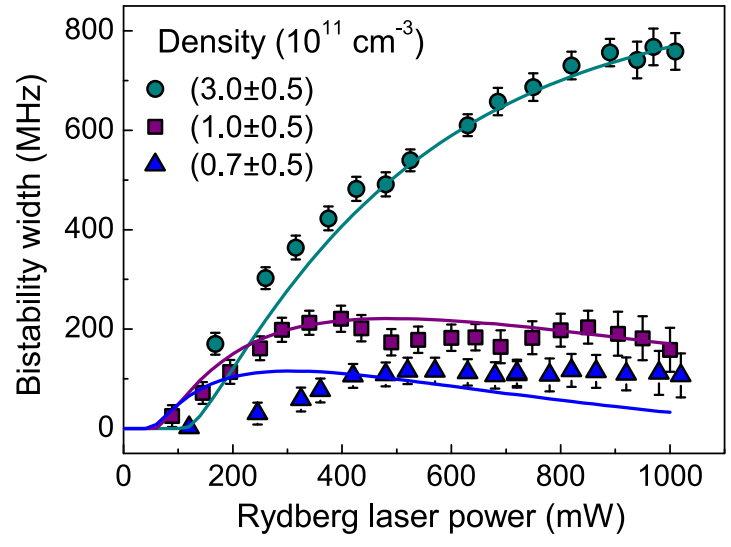

FIG. 5. Bistability width as a function of the Rydberg laser power for $n=28$ and three different atomic densities $N=\{3.0(\mathrm{O})$; $1.0(\square) ; 0.7(\triangle)\} \pm 0.5 \times 10^{11} \mathrm{~cm}^{-3}$. The solid lines are the theoretical calculation using the parameters $\beta^{\prime} / 2 \pi=(1.6 \pm 0.2) \times 10^{-9} \mathrm{~Hz} \mathrm{~cm}^{3}$, $\Gamma_{\text {rg }} / 2 \pi=13.5 \mathrm{MHz}$, and $\Omega / 2 \pi=(137 \pm 2) \sqrt{P} \mathrm{MHz}$, where $P$ is the Rydberg laser power.

power because $V$ is proportional to the atomic density $N$. However, we note that the "low" density response does not fall with Rabi rate as the model predicts. This is an indication that the model does not capture the full behavior of the system. Nevertheless, the data shows good agreement for high density and we are able to describe the behavior of the bistability width.

\section{CONCLUSIONS}

In conclusion, we investigated intrinsic optical bistability in cesium vapor in a multiphoton-excitation scheme to Rydberg states. We characterized the behavior of the bistability width as a function of the principal quantum number and the Rydberg laser power for Rydberg states $n P_{3 / 2}$ with $n$ between 18 and 37 , for different atomic densities. We observed that the shift of the phase transition scales as $n^{4}$ and the width of the hysteresis window exhibits a maximum value with a subsequent narrowing with the increasing of the principal quantum number and a saturation behavior as a function of the Rydberg laser power. A theoretical model using the OBE for a simple two-level system, with modification to include effects of level shifts and broadening, reproduce the behavior of our experimental observation and allows us to estimate broadening and shift coefficients for our phenomenological model. We believe that the observations reported are important for ongoing studies in Rydberg nonlinear optics [32], especially those employing multistep-excitation schemes [33]. The work provides a detailed characterization of systems displaying intrinsic optical bistability and yields insight for theoretical descriptions of such systems. The data presented in this paper are available in Ref. [34].

\section{ACKNOWLEDGMENTS}

This work was supported by Durham University, The Federal Brazilian Agency of Research (CNPq), and EPSRC (Grants No. EP/M014398/1 and No. EP/M013103/1). 
[1] H. Haken, Synergetics, Naturwissenschaften 67, 121 (1980).

[2] M. Marcuzzi, E. Levi, S. Diehl, J. P. Garrahan, and I. Lesanovsky, Phys. Rev. Lett. 113, 210401 (2014).

[3] H. M. Gibbs, Optical Bistability: Controlling Light with Light (Academic Press, Orlando, 1985).

[4] G. I. Stegeman, G. Assanto, R. Zanoni, C. T. Seaton, E. Garmire, A. A. Maradudin, R. Reinisch, and G. Vitrant, Appl. Phys. Lett. 52, 869 (1988).

[5] F. Y. Wang, G. X. Li, H. L. Tam, K. W. Cheah, and S. N. Zhu, Appl. Phys. Lett. 92, 211109 (2008).

[6] Y.-D. Kwon, M. A. Armen, and H. Mabuchi, Phys. Rev. Lett. 111, 203002 (2013).

[7] G. A. Wurtz, R. Pollard, and A. V. Zayats, Phys. Rev. Lett. 97, 057402 (2006).

[8] N. Kravets, A. Piccardi, A. Alberucci, O. Buchnev, M. Kaczmarek, and G. Assanto, Phys. Rev. Lett. 113, 023901 (2014).

[9] H. J. Carmichael and D. F. Walls, J. Phys. B: At. Mol. Phys. 10, L685 (1977).

[10] H. Weimer, Phys. Rev. Lett. 114, 040402 (2015).

[11] N. Šibalić, C. G. Wade, C. S. Adams, K. J. Weatherill, and T. Pohl [Phys. Rev. A (to be published)], arXiv:1512.02123.

[12] M. P. Hehlen, H. U. Gudel, Q. Shu, J. Rai, S. Rai, and S. C. Rand, Phys. Rev. Lett. 73, 1103 (1994).

[13] C. Carr, R. Ritter, C. G. Wade, C. S. Adams, and K. J. Weatherill, Phys. Rev. Lett. 111, 113901 (2013).

[14] C. Carr, M. Tanasittikosol, A. Sargsyan, D. Sarkisyan, C. S. Adams, and K. J. Weatherill, Opt. Lett. 37, 3858 (2012).

[15] C. Carr, C. S. Adams, and K. J. Weatherill, Opt. Lett. 37, 118 (2012).

[16] P. Thoumany, Th. Germann, T. Hänsch, G. Stania, L. Urbonas, and Th. Becker, J. Mod. Opt. 56, 2055 (2009).

[17] A. Gallagher and E. L. Lewis, J. Opt. Soc. Am. 63, 864 (1973).
[18] L. Deng, M. G. Payne, and W. R. Garret, Phys. Rep. 429, 123 (2006).

[19] R. E. Olson, Phys. Rev. Lett. 43, 126 (1979).

[20] W. R. Anderson, J. R. Veale, and T. F. Gallagher, Phys. Rev. Lett. 80, 249 (1998).

[21] G. Lindblad, Commun. Math. Phys. 48, 119 (1976).

[22] T. E. Lee, H. Häffner, and M. C. Cross, Phys. Rev. Lett. 108, 023602 (2012).

[23] T. F. Gallagher, Rydberg Atoms (Cambridge University Press, Cambridge, 1994).

[24] M. Chret, L. Barbier, W. Lindinger, and R. Deloche, J. Phys. B: At. Mol. Phys. 15, 3463 (1982).

[25] B. P. Stoicheff and E. Weinberger, Phys. Rev. Lett. 44, 733 (1980).

[26] A. Reinhard, T. Cubel Liebisch, K. C. Younge, P. R. Berman, and G. Raithel, Phys. Rev. Lett. 100, 123007 (2008).

[27] E. L. Lewis, Phys. Rep. 58, 1 (1980).

[28] L. Weller, R. J. Bettles, P. Siddons, C. S. Adams, and I. G. Hughes, J. Phys. B: At., Mol. Opt. Phys. 44, 195006 (2011).

[29] D. C. Thompson, E. Weinberger, G. X. Xu, and B. P. Stoicheff, Phys. Rev. A 35, 690 (1987).

[30] N. R. de Melo and S. S. Vianna, J. Opt. Soc. Am. B 31, 1735 (2014)

[31] M. A. Mazing and P. D. Serapinas, Zh. Eksp. Teor. Fiz. 60, 541 (1971) [Sov. Phys. JETP 33, 294 (1971)].

[32] J. D. Pritchard, K. J. Weatherill, and C. S. Adams, Ann. Rev. Cold. At. Mol. 1, 301 (2013).

[33] J. M. Kondo, N. Šibalić, A. Guttridge, C. G. Wade, N. R. De Melo, C. S. Adams, and K. J. Weatherill, Opt. Lett. 40, 5570 (2015).

[34] Durham University; the data associated with this work is available at https://collections.durham.ac.uk/files/8c97kq405 